\title{
High spatiotemporal resolution fMRI using partial separability model
}

\author{
Caiyun Shi ${ }^{\mathrm{a}}$, Guoxi Xie ${ }^{\mathrm{a}, \mathrm{b}, *}$, Xiaoyong Zhang ${ }^{\mathrm{a}, \mathrm{c}}$, Shi Su${ }^{\mathrm{a}}$, Yongqin Zhang ${ }^{\mathrm{d}}$, Lijuan Zhang ${ }^{\mathrm{a}}$, \\ Bensheng Qiu ${ }^{\mathrm{c}}$ and Xin Liu ${ }^{\mathrm{a}}$,* \\ ${ }^{a}$ Shenzhen Key Laboratory for MRI, Lauterbur Research Center for Biomedical Imaging, Shenzhen \\ Institutes of Advanced Technology of Chinese Academy of Sciences, Shenzhen, China \\ ${ }^{b}$ Beijing Center for Mathematics and Information Interdisciplinary Sciences, Beijing, China \\ ${ }^{c}$ Centers for Biomedical Engineering, College of Information Science and Technology, \\ University of Science and Technology of China, Hefei, China \\ ${ }^{d}$ School of Information Science and Technology, Northwest University, Xian, China
}

\begin{abstract}
Blood oxygenation level dependent functional MRI (BOLD fMRI) requires repeatedly scanning the same region to capture neuronal activities, so the sampling data is very sparse along the temporal direction, which offers an opportunity to accelerate the fMRI. In this paper, (k-t) space data is sparsely acquired and reconstructed for BOLD fMRI using a partial separability (PS) model with a $\ell_{2}$-norm constraint. The proposed approach achieves a high temporal resolution of $200 \mathrm{~ms}$ without compromising spatial resolution $\left(3.5 \times 3.5 \times 4.0 \mathrm{~mm}^{3}\right)$. A simulation based on the EPI data with the right finger tapping experiment demonstrates that the proposed method can realize high spatiotemporal fMRI with accurate reconstruction of the activation regions from highly undersampled data. Meanwhile, preliminary in vivo experiment results also demonstrate the potential application of the proposed method in fMRI.
\end{abstract}

Keywords: fMRI (function magnetic resonance imaging), partial separability (PS) model, sparse sampling, BOLD

\section{Introduction}

Functional magnetic resonance imaging(fMRI), which is a technique that measures the hemodynamic response related to neural activity in the brain, requires a high spatial and temporal resolution [1]. Echo-planar imaging (EPI) provides a scan time of tens of milliseconds for a slice imaging and a spatial resolution of 3-4 mm. In addition, other significant efforts based on EPI have been made to accelerate data acquisition for fMRI using fast scanning $[2,3]$ and/or parallel imaging $[4,5]$. However, the

\footnotetext{
Address for correspondence: Guoxi Xie, Shenzhen Key Laboratory for MRI, Lauterbur Research Center for Biomedical Imaging, Shenzhen Institutes of Advanced Technology of Chinese Academy of Sciences, Shenzhen, China. Tel.: +86-0755-86392287; Fax: +86-0755-86392245; Email: gx.xie@siat.ac.cn.

Xin Liu, Paul C. Lauterbur Research Center for Biomedical Imaging, Institute of Biomedical and Health Engineering, Shenzhen Institutes of Advanced Technology, Chinese Academy of Sciences, Tel.: +86-0755-86392287; Fax: +86-0755-86392299; Email: xin.liu@siat.ac.cn.
} 
resolution of an fMRI image based on this fast scanning is limited by technological challenges [6]. A sparse sampling model provides a new way for the high spatial and temporal resolution fMRI. One of the higher-order generalized series imaging with a parallel imaging technique has been introduced for highspatial resolution fMRI [7, 8]. However, disadvantages of this method include the limitation on the number of imaging slices, and the reduced number of encode lines. As a result, the quality of the reconstructed image is generally degraded. Other sparse sampling models include the partial separability (PS) $[9,10]$ and compressed sensing (CS) [11-14]. Holland et al shows that the $\ell_{1}$-norm based CS reconstruction can produce an increase in functional contrast and yield equivalent fMRI sensitivity when applied to $28 \%$ under-sampled data [13]. However, the spatial and temporal resolutions of BOLD fMRI are tradeoffs using the CS-based method. In this work, a dynamic model of partial separability (PS) with $\ell_{2}$-norm constraint was introduced to improve the temporal resolution of BOLD fMRI from $2000 \mathrm{~ms}$ to $200 \mathrm{~ms}$ without compromising the spatial resolution. As the temporal resolution of BOLD fMRI improved, the increase in the time frame may lead to more statistical signal power compared to the fully sampled acquisition.

\section{Theory}

The $L t h$-order PS model represents the spatiotemporal image function as Eq. (1)

$$
\rho(r, t)=\sum_{\ell=1}^{L} \mathrm{~V}_{\ell}(t) U_{\ell}(r)
$$

where $U_{\ell}(r)\left(U \in C^{N \times L}\right)$ and $\mathrm{V}_{\ell}(t)\left(V \in C^{M \times L}\right)$ are the $\ell$ th spatial and temporal basis functions, respectively. $L$ is the model order, $N$ is the pixel number, and $M$ is the image number. Our purpose is to recover image $\rho(r, t)$ from a sub-Nyquist measured data $d(k, t)$, which is expressed as Eq. (2) in matrix form.

$$
d=\Omega \mathrm{F}(V U)+\xi
$$

where $F$ is the Fourier operator that transforms the image into k space, $\Omega$ is the undersampling matrix, and $\xi$ is white Gaussian noise. Generally, the temporal basis $V$ can be obtained by applying the singular value decomposition (SVD) method [10] on a training dataset that is acquired along with the measured data. Thus, the least-squares method can be used to solve Eq. (2) and get the spatial basis $U$. Because the measured data is highly undersampled, directly solving Eq. (2) without any constraint would result in noise amplification and potential image artifacts. As the BOLD signal changes little $(\sim 3 \%)$, a $\ell_{2}$ -norm constraint was used to suppress the noise for solving Eq. (2), which can be described as Eq. (3)

$$
\hat{U}=\arg \min _{U \in C^{L \times N}}\|d-\Omega \mathrm{F}(V U)\|_{2}^{2}+\lambda\|U\|_{2}^{2}
$$

where $\lambda$ is the regularization parameter [15]. The first term in the right side of Eq. (3) is the data 
consistency and the second one is the $\ell_{2}$-norm constraint. The solution of Eq. (3) can be written exactly as Eq. (4) which can be quickly evaluated using a conjugate gradient algorithm [16].

$$
\left(V^{H} F^{H} \Omega^{H} \Omega F V+\lambda I\right) U=V^{H} F^{H} \Omega^{H} d
$$

where $H$ is Hermitian transpose.

\section{Materials and methods}

\subsection{Numerical simulations}

The proposed method was evaluated using an experimental fMRI dataset that was acquired by a 2D EPI sequence on a 3T MRI (SIEMENS Tim Trio, Germany). A periodic block design paradigm (Figure 1) with the right finger tapping action was performed for collecting the data. In the experiment, a healthy volunteer who participated in the study had not experienced any medical, neurological, or psychiatric illnesses. The study was given ethical approval by the appropriate Institutional Review Boards and written informed consent was obtained from the study participant prior to MR scan. The fMRI data was then acquired using a 2D EPI sequence with the parameters including: TR/TE $=2000 / 30 \mathrm{~ms}$, matrix size $=64 \times 64$, slice thickness $=3.5 \mathrm{~mm}$ with $35 \%$ gaps, and 32 slices. The field of view was $(F O V)=220 \mathrm{~mm}$ $\times 220 \mathrm{~mm}$, and the flip angle $=80^{\circ}$. The acquired data was then treated as a reference and used to simulate the undersampled data for PS model as illustrated in Figure 2. The repeatedly sampled phase encodings near the center of k-space is referred to as training data $S_{\text {Train }}(k, t)$, which is used to estimate the temporal basis $V$ [17-19]). The image data $S_{I m g}(k, t)$, which is the measured data $d(k, t)$ in Eq. (2), was collected by sparse sampling the phase encodings of the referred k-space. After the training and image data were collected, image reconstruction was performed using the proposed method in Eq. (3).

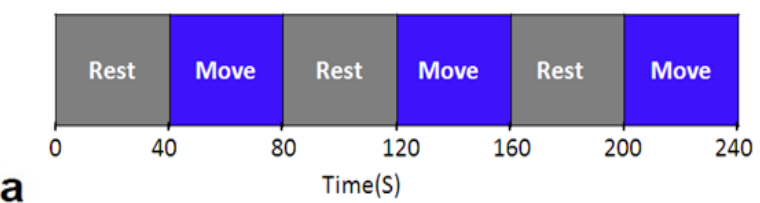

a
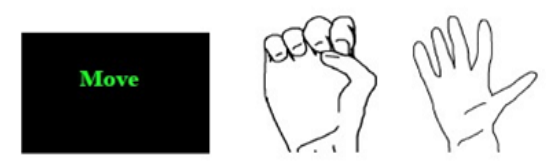

b
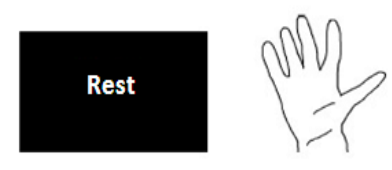

Fig. 1. The stimulus mode for the fMRI experiment.



Fig. 2. An illustration of the proposed data acquisition scheme. 

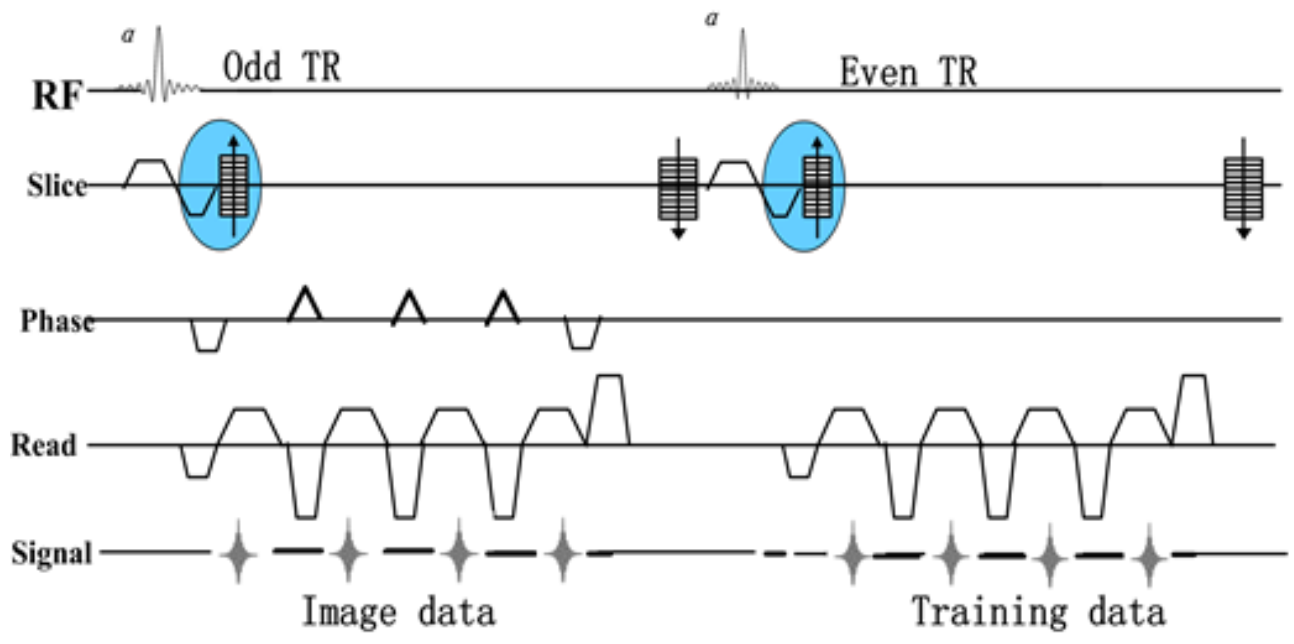

Fig. 3. A schematic diagram of the customized sequence.

\subsection{In vivo study}

An in vivo experiment with the same finger tapping task was performed on the same 3T MRI scanner using a customized sequence (see Figure 3). The training data and the image data were acquired for image reconstruction using the proposed method. Scan parameters included: TR/TE $=100 / 20 \mathrm{~ms}$, bandwidth $=880 \mathrm{~Hz} /$ pixel, flip angle $=10^{\circ}$, and 3D data acquisition fashion with spatial resolution $3.5 \times 3.5 \times 4.0 \mathrm{~mm}^{3}$. Because training data determined the temporal resolution and the image data determined the spatial resolution, a BOLD fMRI with a temporal resolution of $200 \mathrm{~ms}$ and a spatial resolution of $3.5 \times 3.5 \times 4.0 \mathrm{~mm}^{3}$ were achieved using the proposed method.

The training data and the image data were interleaved acquired for the proposed method.

\section{Results and discussion}

\subsection{Simulation results}

The EPI-based data demonstrated the ability of high temporal resolution fMRI. Figure 4 shows two representative reconstruction images of the proposed method and EPI. It is clear that the proposed PS method could effectively suppress noise. Figure 5 shows representative neuronal activation maps from the proposed method. The neuronal activation map reconstructed by the proposed method was analyzed with SPM (http://www.fil.ion.ucl.ac.uk/spm/), and the activation regions were overlaid on the corresponding reconstructed images. The neuronal activation reconstructed by the proposed method matched well with that reconstructed by of the reference EPI data. The BOLD signal variation was also consistent with the reference results (Figure 6).

\subsection{In vivo experiment results}

In vivo experiment results are shown in Figures 7 and 8. A much higher temporal resolution of 200ms was achieved without compromising spatial resolution. Compared with the conventional PS model without regularization, the noise was suppressed by the proposed method (Figure 7). As the noise 
suppressed, the neuronal activation can be found after the reconstructed images are processed by SPM [20]. It should be noted that the location of neuronal activation using the proposed method (Figure 8) was found to be different with the reference (Figure 5), which needs to be further evaluated and studied.
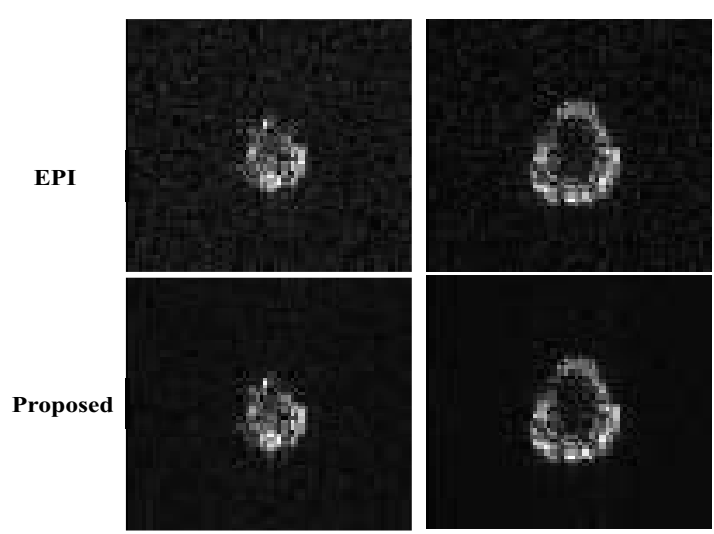

Fig. 4. The reconstruction images of the proposed method and EPI.

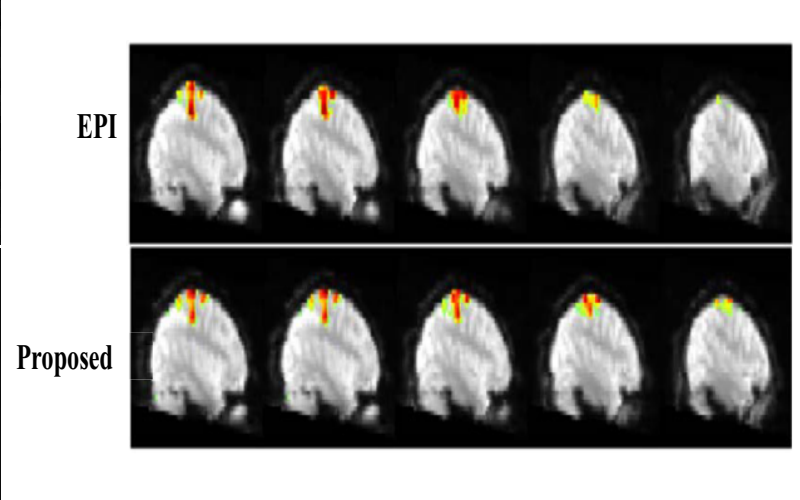

Fig. 5. Representative neuronal activation maps from the fully sampled data with those from the proposed method (Sagittal).

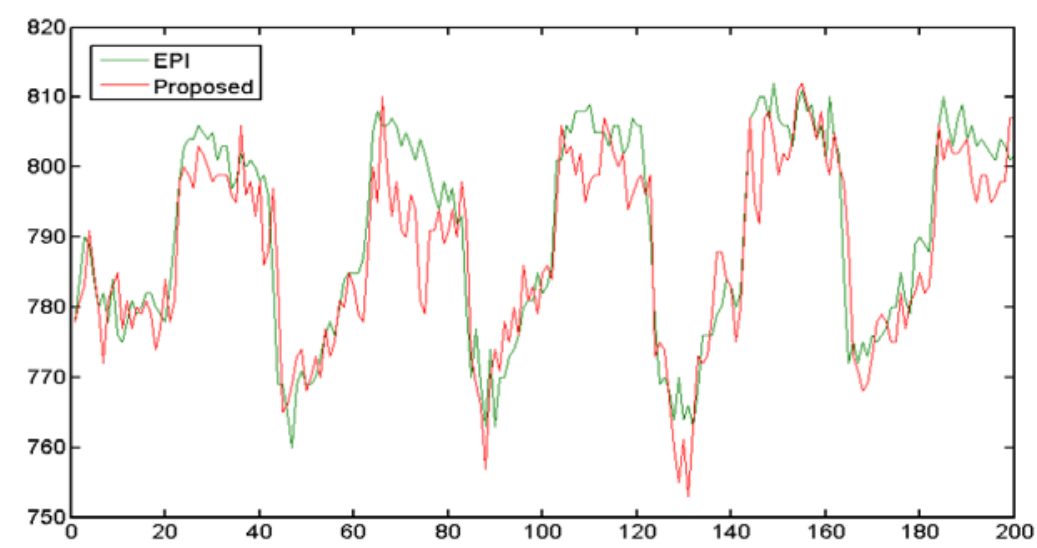

Fig. 6. BOLD signal variation curves. The activation result of proposed method (red curve) matched well with that of the reference EPI (green curve).

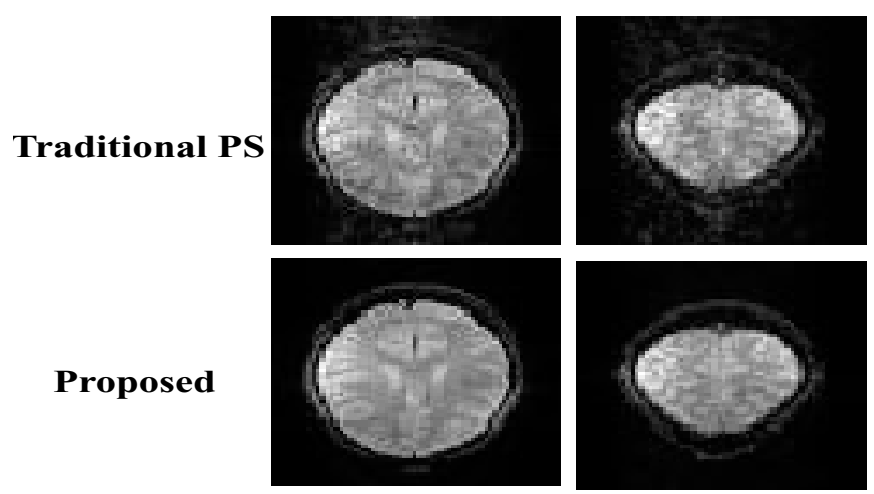

Fig. 7. The fMRI images of our proposed method compared with traditional PS model. 



Fig. 8. Representative neuronal activation maps of the proposed method with a traditional PS model. Compared to the proposed method, the location of neuronal activation of the traditional PS changed due to the noise amplification; the traditional PS model achieved poor functional imaging results.

\section{Discussion}

In this study, the PS model was used for a high spatiotemporal fMRI. Generally, EPI-based methods with parallel imaging techniques are used to achieve a sufficient imaging speed in fMRI studies. However, the parallel imaging technique causes SNR degradation [21]. For example, the SENSE (sensitivity encoding) technique with an acceleration factor of $R$ leads to the SNR reduction of a factor of $\mathrm{g} \sqrt{R}$, where $\mathrm{g}$ indicates the coil geometry factor. Sparse sampling offers a new way to further accelerate fMRI. Recently, the two basic sparse sampling imaging methods, including compressed sensing (CS) and a partial separability (PS) model, have been used to improve the spatiotemporal resolution of MR imaging. The PS model overcomes the tradeoff of the spatial and temporal resolutions of BOLD fMRI, but the tradeoff still existed for CS-based methods. However, the undersampling of $\mathrm{k}$ space data based on the PS model often causes noise amplification and potential image artifacts. It is therefore useful to regularize the model by incorporating additional penalties. In this paper, a $\ell_{2}$-norm penalty to the spatial basis $\|\mathrm{U}\|_{2}$ was used to suppresses the noise for image reconstruction, which could realize more accurate brain activation mapping and high-resolution functional images.

In the in vivo experiment, the efficiency of undersampled fMRI acquisition schemes illustrated that the proposed method achieved a much higher temporal resolution compared to the EPI. However, as shown in Figure 8, the location of neuronal activation using the proposed method was found to be different with the reference. Further evaluation and a large-scale subject study are necessary for our future work.

\section{Conclusion}

This paper discusses a novel method based on the PS model for accelerating fMRI experiments using sparse sampling of $(\mathrm{k}, \mathrm{t})$-space data. The performance of the proposed method has been preliminarily evaluated using a retrospective undersampling of an EPI-based fMRI dataset and an in vivo healthy volunteer study. The simulation results demonstrated that the proposed method could accurately 
reconstruct the magnitude images with much higher temporal resolution and capture the BOLD signal change for fMRI study. The in vivo experiment results also demonstrated the feasibility of the proposed method. However, further evaluation and optimization of the proposed method need to be carried out in the future.

\section{Acknowledgment}

This work was supported in part by the National Basic Research Program of China (973 Program) (No. 2015CB755500), the National Natural Science Foundation of China (No. 81000611, No. 61201442), the International Cooperation and Exchange of the National Science Foundation of China (No.81120108012, No.81328013), and the Natural Science Foundation of Shenzhen (No. JCYJ20140417113430603) and Guangdong (No. 2014A030310360).

\section{References}

[1] S. Ogawa, T. Lee, A. Kay and D. Tank, Brain magnetic resonance imaging with contrast dependent on blood oxygenation, Proceedings of the National Academy of Sciences 87 (1990), 9868-9872.

[2] D.A. Feinberg, S. Moeller, S.M. Smith, E. Auerbach, S. Ramanna, M.F. Glasser, K.L. Miller, K. Ugurbil and E. Yacoub, Multiplexed echo planar imaging for sub-second whole brain FMRI and fast diffusion imaging, PloS One 5 (2010), e15710-e15720.

[3] G. Liu, G. Sobering, J. Duyn and C.T. Moonen, A. functional MRI technique combining principles of echo-shifting with a train of observations (PRESTO), Magnetic Resonance in Medicine 30 (1993), 764-768.

[4] O. Afacan, W.S. Hoge, F. Janoos, D.H. Brooks and I.A. Morocz, Rapid full-brain fMRI with an accelerated multi shot 3D EPI sequence using both UNFOLD and GRAPPA,Magnetic Resonance in Medicine 67 (2012), 1266-1274.

[5] J.A. de Zwart, P. van Gelderen, P. Kellman and J.H. Duyn, Application of sensitivity-encoded echo-planar imaging for blood oxygen level-dependent functional brain imaging, Magnetic Resonance in Medicine 48 (2002), 1011-1020.

[6] F.H. Lin, T.Y. Huang, N.K. Chen, F.N. Wang, S.M. Stufflebeam, J.W. Belliveau, L.L. Wald and K.K. Kwong, Functional MRI using regularized parallel imaging acquisition, Magnetic Resonance in Medicine 54 (2005), 343-353.

[7] S. Yun, S.S. Oh, Y. Han and H. Park, High-resolution fMRI with higher-order generalized series imaging and parallel imaging techniques (HGS-parallel), Journal of Magnetic Resonance Imaging 29 (2009), 924-936.

[8] H.M. Nguyen and G. Glover, A modified generalized series approach: Application to sparsely-sampled fMRI, IEEE Transactions on Biomedical Engineering 60 (2013), 2867-2877.

[9] F. Lam, B. Zhao, Y. Liu, Z.-P. Liang, M. Weiner and N. Schuff, Accelerated fMRI using low-rank model and sparsity constraints, International Society for Magnetic Resonance in Medicine, 2013, 2620.

[10] Z.-P. Liang, Spatiotemporal imaging with partially separable functions, IEEE International Symposium on Biomedical Imaging, 2007, 988-991.

[11] H. Jung, K. Sung, K.S. Nayak, E.Y. Kim and J.C. Ye, k-t FOCUSS: A general compressed sensing framework for high resolution dynamic MRI, Magnetic Resonance in Medicine 61 (2009), 103-116.

[12] O. Jeromin, M.S. Pattichis and V.D. Calhoun, Optimal compressed sensing reconstructions of fMRI using 2D deterministic and stochastic sampling geometries, Biomedical Engineering Online 11 (2012), 1-36.

[13] D. Holland, C. Liu, X. Song, E. Mazerolle, M. Stevens, A. Sederman, L. Gladden, R. D'Arcy, C. Bowen and S. Beyea, Compressed sensing reconstruction improves sensitivity of variable density spiral fMRI, Magnetic Resonance in Medicine 70 (2013), 1634-1643.

[14] H. Jung and J.C. Ye, Performance evaluation of accelerated functional MRI acquisition using compressed sensing, IEEE International Symposium on Biomedical Imaging, 2009, 702-705.

[15] C. Brinegar, H. Zhang, Y.-J. Wu, L.M. Foley, T.K. Hitchens, Q. Ye, D. Pocci, F. Lam, C. Ho and Z.-P. Liang, Real-time cardiac MRI using prior spatial-spectral information, Annual International Conference of the Engineering in Medicine and Biology Society, 2009, 4383-4386.

[16] Y.-H. Dai and C.-X. Kou, A nonlinear conjugate gradient algorithm with an optimal property and an improved Wolfe line search, SIAM Journal on Optimization 23 (2013), 296-320. 
[17] G.-X. Xie, Z. Weng, C. Zou, X. Feng, H.-R. Zheng, X. Liu and B. Qiu, A simulation study of dynamic MRI based on partially separable functions and keyhole techniques, 4th International Conference on Biomedical Engineering and Informatics (BMEI) 1 (2011), 68-72.

[18] J.P. Haldar and Z.-P. Liang, Spatiotemporal imaging with partially separable functions: A matrix recovery approach, IEEE International Symposium on Biomedical Imaging, 2010, 716-719.

[19] B. Zhao, J.P. Haldar, A.G. Christodoulou and Z.-P. Liang, Image reconstruction from highly undersampled-space data with joint partial separability and sparsity constraints, IEEE Transactions on Medical Imaging 31 (2012), 1809-1820.

[20] K.J. Friston, A.P. Holmes, K.J. Worsley, J.P. Poline, C.D. Frith and R.S. Frackowiak, Statistical parametric maps in functional imaging: A general linear approach, Human Brain Mapping 2 (1994), 189-210.

[21] J.A. de Zwart, P. van Gelderen, X. Golay, V.N. Ikonomidou and J.H. Duyn, Accelerated parallel imaging for functional imaging of the human brain, NMR in Biomedicine 19 (2006), 342-351. 